\title{
GEOMETRIA DINÂMICA EM TABLETS: ESTUDO DE CASO COM O APLICATIVO GEOGEBRA
}

\author{
Larissa de Sousa Moreira, IF Fluminense Campus-Itaperuna \\ larissasm@iff.edu.br \\ Gilmara Teixeira Barcelos Peixoto, IF Fluminense Campus Campos-Centro \\ gilmarab@iff.edu.br \\ Silvia Cristina Freitas Batista, IF Fluminense Campus Campos-Centro \\ silviac@iff.edu.br
}

\section{RESUMO}

Recursos para Geometria Dinâmica favorecem práticas pedagógicas investigativas. Assim, é importante que a formação de professores contemple o uso dos mesmos. Nesse contexto, este artigo visa analisar um estudo de caso promovido na Licenciatura em Matemática de uma instituição federal, envolvendo o uso do software GeoGebra no estudo de Geometria Plana. O objetivo da pesquisa foi captar a visão dos licenciandos sobre o aplicativo GeoGebra para tablets Android, promovendo uma comparação com a versão para computador. Neste artigo, discute-se, inicialmente, o uso pedagógico de tablets e caracteriza-se o GeoGebra. A seguir, são descritos os procedimentos metodológicos adotados e são discutidos os resultados encontrados. Em geral, a versão do GeoGebra para tablets foi avaliada positivamente pelos licenciandos, mas, em alguns aspectos o uso da versão no computador foi considerado mais fácil.

Palavras-chave: GeoGebra, tablets, Geometria Dinâmica

\section{DYNAMIC GEOMETRY USING TABLETS: A CASE STUDY WITH THE APPLICATION GEOGEBRA}

\begin{abstract}
Resources for Dynamic Geometry favor investigative teaching practices. Thus, it is important that teacher education contemplates the use of these resources. In this scenario, this article aims at analyzing a case study conducted at a federal institution with students of Math Education, focusing on the use of GeoGebra software in the study of Plane Geometry. The objective of the study was to verify the students' perception of the GeoGebra app for Android tablets compared to the software version for traditional computers. The paper discusses the pedagogic use of tablets and describes characteristics of GeoGebra. This is followed by a description of the methodological procedures and the analysis of data obtained in the study. The GeoGebra app for tablets received good evaluation by students, but the traditional version was considered easier in some aspects.
\end{abstract}

Keywords: GeoGebra, tablets, Dynamic Geometry

\section{Introdução}

As tecnologias digitais (TD) e as mídias sociais têm contribuído para mudanças na vida cotidiana das pessoas, transformando as formas de entretenimento e de aprendizagem, entre outros aspectos (Nascimento, 2013). É importante ressaltar, no entanto, que apenas a inclusão de TD em escolas e a disponiblização de conteúdos na rede não garantem mudanças positivas no processo de ensino e aprendizagem. O momento e a 
forma como os professores adotam tecnologias são aspectos que influenciam, diretamente, na ocorrência, ou não, de melhorias nesse processo. Portanto, os professores são pontos chave do sistema educacional. $\mathrm{O}$ benefício alcançado com o uso de TD em sala de aula depende, entre outros aspectos, do preparo desses profissionais (Nascimento, 2013).

Neste contexto, é de suma importância que ocorram iniciativas que contemplem a formação inicial e continuada para a integração das TD ao contexto escolar. Uma perspectiva teórica que pode fundamentar essa integração é o Technological Pedagogical Content Knowledge (TPACK), ou seja, conhecimento tecnológico, pedagógico e do conteúdo (Koehler; Mishra, 2009). Esta teoria baseia-se no conceito de saber pedagógico, desenvolvido por Shulman (1986). Define-se TPACK como o conhecimento que os professores necessitam ter para ensinar com e sobre tecnologias nas diversas áreas do conhecimento, incluindo a discussão de questões pedagógicas sobre o uso das TD no estudo de conteúdos (Koehler; Mishra, 2009).

Considerando a teoria TPACK e as potencialidades das TD no processo de ensino e aprendizagem de Matemática, foi realizado um estudo de caso na disciplina Educação Matemática e Tecnologias da Licenciatura em Matemática de uma instituição federal, em setembro de 2013. O objetivo do estudo foi captar a visão dos licenciandos sobre o aplicativo GeoGebra para tablets Android, promovendo uma comparação com a versão para computador. Entre outras ações, foram propostas atividades que contemplam temas de Geometria Plana.

Assim, este artigo tem por objetivo apresentar a percepção dos licenciandos quanto ao uso pedagógico do Geogebra no computador e no tablet. Para tanto, na seção 2, é abordado o uso pedagógico de tablets. Na seção 3, caracteriza-se o software GeoGebra. $\mathrm{Na}$ seção 4, são descritos os procedimentos metodológicos adotados no estudo de caso e, na seção 5, são discutidos os resultados encontrados. Finalizando, na seção 6, são apresentadas considerações sobre o estudo promovido.

\section{Uso Pedagógico de Tablets}

Em todo o mundo, diversos países estão experimentando o uso de tablets na educação formal (Clarke et al., 2013). Segundo os autores, tablets podem ser vistos como caixas portáteis de ferramentas pedagógicas. Algumas dessas ferramentas são apropriadas para registro, organização e revisão de informações, outras podem contribuir em atividades mais complexas, envolvendo, por exemplo, o desenvolvimento do pensamento crítico, da autoconfiança e da habilidade de apresentação. Em geral, o uso desses dispositivos contribui para a motivação e o envolvimento dos alunos. Além disso, o professor possui mais recursos para lidar com as diferenças individuais de aprendizagem (Clarke et al., 2013). Também destacando o potencial pedagógico dos tablets, Seabra (2012) afirma que os mesmos oferecem um conjunto de recursos que podem facilitar a visualização de conteúdos, estimular atividades cooperativas e o desenvolvimento de projetos e, assim, contribuir para a realização de diversas atividades pedagógicas.

Em um estudo de caso sobre o uso de tablets na educação, Ciampa (2013) relata as percepções da professora e dos alunos, em relação às potencialidades motivacionais do uso desses dispositivos para a aprendizagem. O estudo foi promovido no Canadá e, no mesmo, uma professora do Ensino Fundamental utilizou tablets com dez de seus alunos, em atividades pedagógicas. Os quizzes e jogos utilizados, segundo o relato, proporcionaram oportunidades de feedback imediato e auto-avaliação. A professora e a maioria dos alunos participantes consideraram que tais possibilidades incentivaram o desenvolvimento das tarefas propostas. A interatividade e o feedback automático possibilitados pelo tablets também podem ter contribuído para o uso voluntário dos aplicativos fora da sala de aula e para o aumento da curiosidade cognitiva dos alunos. 
Goodwin (2012) também aponta vantagens do uso pedagógico de tablets. O autor relata uma pesquisa realizada em três escolas da Austrália, em 2011, envolvendo cinco professores, mais de 90 estudantes e 75 iPads. Os resultados sinalizaram que o uso desses dispositivos pode melhorar o engajamento, a motivação dos alunos e a colaboração entre os mesmos, tanto presencialmente quanto a distância. Além disso, pode contribuir para aprendizagens mais personalizadas e para a melhoria de resultados educacionais. Os professores atribuíram esses ganhos a vários fatores: i) portabilidade do aparelho; ii) habilidade dos professores para lidar com necessidades e preferências pessoais; iii) facilidade com que os alunos utilizavam aplicativos e ferramentas; iv) adoção da concepção de que o tablet era uma ferramenta de aprendizagem.

Certamente, apesar de todas as possibilidades pedagógicas dos tablets, alguns aspectos merecem cuidados. Clarke et al. (2013), por exemplo, destacam que é importante considerar que os alunos adotam, em geral, uma postura multitarefa, utilizando vários recursos, simultaneamente, o que pode gerar distrações. Nesse sentido, é importante ter regras claras. A forma de lidar com essa situação também depende do quanto os professores estão dispostos a abrir mão do controle da sala de aula (Clarke et al., 2013). Seabra (2012), por sua vez, destaca que o uso pedagógico dos tablets irá requerer um professor preparado, dinâmico e investigativo.

Mang e Wardley (2012) apresentam recomendações para o uso de tablets, a partir de uma experiência com alunos do Ensino Superior, usando iPads. Segundo os autores, é fundamental que os dispositivos sejam utilizados, regularmente, nas atividades didáticas, para que o uso se torne natural. Além disso, recomendam, entre outras coisas, que os professores conheçam bem o tablet, antes de utilizá-lo em sala de aula, e planejem, adequadamente, as atividades pedagógicas.

Considerando a importância de papel do professor em todo esse contexto, Kearney e Maher (2013) descrevem uma pesquisa promovida no âmbito de um projeto australiano denominado Teaching Teachers for the Future. A pesquisa envolveu 16 alunos do Bacharelado em Educação, cursando uma disciplina de Educação Matemática, e dois professores dessa disciplina. $\mathrm{Na}$ pesquisa, foram utilizados Ipads em atividades de aprendizagem, realizadas dentro e fora de sala de aula, com o objetivo de investigar como as tecnologias móveis poderiam apoiar a aprendizagem profissional. Fenômenos matemáticos foram capturados em situações cotidianas, fora de sala de aula, e discutidos, posteriormente, em termos de atividades formais. O uso de iPads também contribuiu para aumentar a produtividade e a capacidade de registrar informações. No entanto, houve pouca colaboração em rede (conversas e compartilhamento de dados), embora os licenciandos valorizassem as possibilidades do dispositivo para tal fim.

Assim, de acordo com o que foi discutido nesta seção, observa-se que os tablets têm potencial para apoiar atividades pedagógicas diversas. Certamente, alguns cuidados são necessários, dentre os quais a formação adequada do professor. Considerando esse contexto, foi realizado o estudo de caso relatado neste artigo, promovido na licenciatura em Matemática, utilizando o software GeoGebra (em computador e tablet). Tal recurso é caracterizado na seção seguinte.

\section{GeoGebra: características gerais}

O GeoGebra ${ }^{1}$ é um software de Matemática Dinâmica, que permite o estudo de Geometria, Álgebra, e Cálculo, além de apresentar características de um CAS (Computer Algebra System), para todos os níveis de ensino (Hohenwarter M.; Preiner, 2007). Trata-se de um programa livre, gratuito, multiplataforma, disponível em vários idiomas, dentre os quais o português. O desenvolvimento do GeoGebra teve origem no mestrado de Markus Hohenwarter (University of Salzburg - Austria), em 2002 (Hohenwarter M., 2013a). 
Desenvolvido para ser utilizado por usuários sem conhecimentos de informática avançada e de forma intuitiva (Hohenwarter M., 2006), o GeoGebra tem por ideia básica oferecer duas representações de cada objeto: a geométrica e a algébrica (Hohenwarter M.; Preiner, 2007). A possibilidade de alterar um objeto por meio dessas duas representações é chamada de Conexão Bidirecional e é o que diferencia o GeoGebra de outros softwares de Geometria Dinâmica e CAS (Hohenwarter M.; Preiner, 2007).

As versões do GeoGebra atualmente disponíveis para computador/notebook são: GeoGebra Desktop e GeoGebraWeb. O GeoGebra Desktop inclui uma versão para download (Windows, Linux e Mac OS) e a versão Java Webstart ${ }^{2}$, que armazena todo o aplicativo localmente, na memória cache do computador. Toda vez que o GeoGebra Java Webstart é iniciado, o mesmo verifica se há uma nova versão disponível. Há, também, a versão 5.0, ainda em teste, por enquanto na forma Java Webstart, que permite construções em 3D.

GeoGebraWeb é uma versão usada diretamente do navegador Web do computador, sem necessidade de plugins. A mesma é semelhante às versões GeoGebra Desktop, reunindo recursos para estudo de diversos tópicos matemáticos, em um único ambiente. Mas, além disso, permite abrir e salvar arquivos no Google Drive e inserir imagem da Webcam, o que não é possível nas versões Desktop. Assim, sem requerer procedimentos de instalação e atualização, o GeoGebraWeb se torna especialmente simples de ser utilizado em redes de computadores de escolas.

Porém, o GeoGebraWeb ainda não possibilita imprimir arquivos diretamente da mesma, nem gerar planilhas dinâmicas, ações possíveis nas versões GeoGebra Desktop. As referidas planilhas são materiais didáticos interativos, que podem ser acessados de qualquer computador, mesmo sem ter o software instalado, necessitando apenas de um navegador e do plugin Java (Preiner, 2008). Muitos professores têm incentivado a aprendizagem experimental e por descoberta, por meio dessas planilhas (Hohenwarter M.; Lavicza, 2007). Assim, o GeoGebra é, também, uma ferramenta para criação de conteúdos e-learning interativos (Hohenwarter M.; Fuchs, 2004).

As planilhas dinâmicas podem ser disponibilizadas em uma plataforma de compartilhamento denominada GeoGebraTube ${ }^{3}$. O usuário cadastrado na plataforma pode compartilhar, além de planilhas dinâmicas, ferramentas criadas no software, coleção de planilhas, vídeos, tutoriais e outros. A plataforma suporta o upload direto de contruções feitas no GeoGebra, permite a pesquisa de materiais, a avaliação de usuários cadastrados e o envio de comentários (Hohenwarter M., 2013a).

Em 2013, foi oficialmente lançada a versão para tablets - Windows 8, Android e iPad (Hohenwarter M., 2013b). A Figura 1 mostra a interface do aplicativo, capturada a partir de um tablet Android. Sendo uma versão simplificada e muito recente, o GeoGebra para tablets ainda não apresenta todas as possibilidades das versões desktop. Uma diferença significativa é o fato de que, nas versões desktop, as ferramentas, além do ícone, apresentam o nome por extenso e uma breve descrição de uso da ferramenta. No GeoGebra para tablets, são apresentados apenas os ícones das ferramentas.

Markus Hohenwarter (2013b) destaca os principais recursos da versão para tablets: i) pesquisa integrada com o GeoGebraTube; ii) visualização de planilhas dinâmicas, a partir do GeoGebraTube; iii) barra de ferramentas e de estilo otimizadas por toque; iv) completa compatibilidade com as versões desktop.

No aplicativo, é possível salvar as construções, mas, não é possível criar planilhas dinâmicas. Para gerar uma dessas planilhas, a partir de uma construção salva no aplicativo Android, é preciso, inicialmente, compartilhar a construção via e-mail, a seguir, salvá-la no computador e, então, disponibilizá-la na plataforma GeoGebraTube. 
No aplicativo, é possível acessar as planilhas disponibilizadas no GeoGebraTube, por meio da ferramenta de pesquisa ou diretamente no navegador.

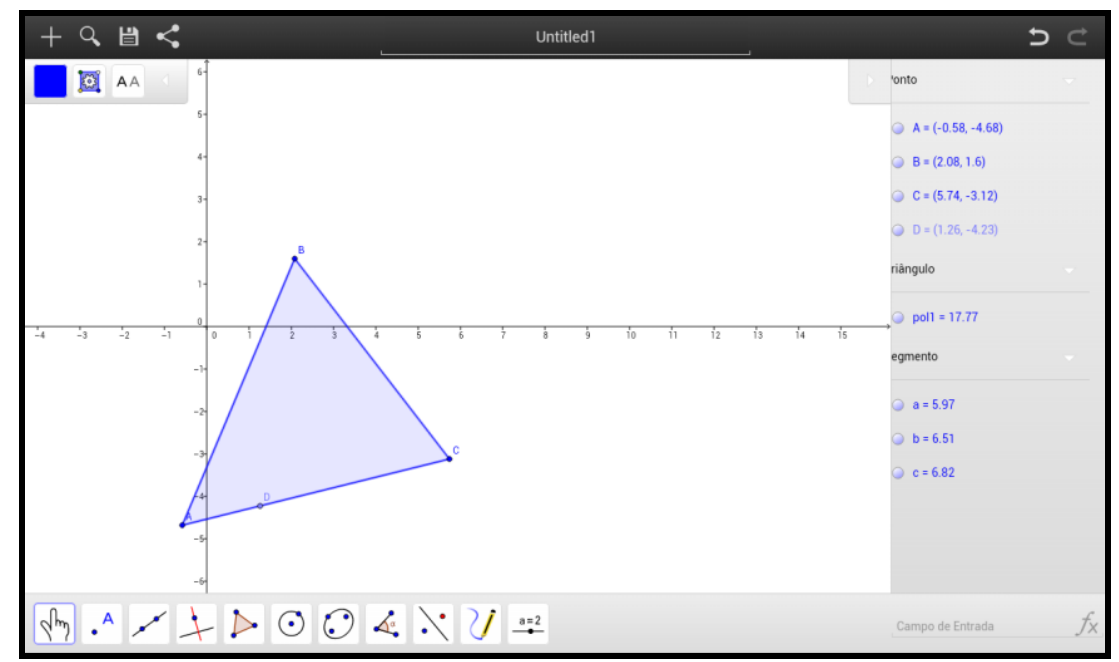

Figura 1: Interface do GeoGebra para tablets Android - versão 4.3.27.1

Segundo Markus Hohenwarter e Fuchs (2004), o GeoGebra pode ser usado em diversos casos: i) para demonstração e visualização; ii) como ferramenta de construção; iii) para a aprendizagem por descoberta; iv) para a preparação de materiais didáticos.

A utilização do GeoGebra tem sido analisada em diversos estudos (Edwards e Jones, 2006; Hohenwarter J. et al., 2008; Zakaria e Lee, 2012; Ljajko, 2013). Em geral, tais estudos apontam que: i) professores e alunos consideram o software fácil de usar e útil em todos os níveis de ensino; ii) o uso do software possibilita a atuação do professor como facilitador da aprendizagem, uma vez que ajuda a diversificar os métodos de ensino; iii) proporciona a criação de materiais didáticos de forma rápida e fácil.

Chaurasia (2013) destaca que o GeoGebra passou a ser visto pelos professores como um ambiente de aprendizagem com potencial para contribuir para o desenvolvimento de diversas habilidades dos alunos. No entanto, Ljajko (2013) alerta que, para obter bons resultados, é preciso fazer uso do software de forma significativa, contar com a dedicação dos professores para construir planilhas criativas e incentivar os estudantes a trabalhar de forma independente, para que não ocorra apenas uma mera reprodução de práticas tradicionais. Nesse mesmo sentido, Preiner (2008) destaca que os materiais didáticos devem priorizar o conteúdo matemático e não o uso do software.

\section{Procedimentos Metodológicos}

Dadas às características da pesquisa, optou-se por realizar um estudo de caso. O mesmo foi promovido na turma do primeiro período da Licenciatura em Matemática de uma instituição federal, na disciplina Educação Matemática e Tecnologias, ministrada por uma das autoras deste artigo. Nessa disciplina, os softwares são estudados segundo o referencial teórico TPACK. Assim, temas matemáticos são abordados com o auxílio de $\mathrm{TD}$, de forma que o uso dessas tecnologias nunca tenha um fim em si mesmo.

O tema matemático selecionado para o estudo de caso foi Geometria Plana, devido às diversas ferramentas para esse fim presentes no GeoGebra. Além disso, os licenciandos estavam cursando a disciplina Geometria I, o que favorecia o entendimento das atividades propostas e da importância do software para a resolução das mesmas.

Os tablets utilizados na pesquisa pertencem ao Projeto Pró-Docência ${ }^{4}$, vinculado à instituição de ensino em questão, e foram adquiridos com verba da CAPES. São sete tablets Motorola XOOM, com sistema operacional Android e tela de 10.1 polegadas. Os mesmos são utilizados em ações com professores em formação, tendo em vista $o$ 
levantamento de potencialidades e dificuldades de uso pedagógico.

Participaram do estudo 21 alunos. Na disciplina em que o experimento ocorreu, a turma é dividida em dois grupos (grupo 1 e grupo 2) que participam das aulas em dias distintos para melhor uso das TD. Este fato permitiu o trabalho com os sete tablets institucionais. Para tanto, os 10 participantes do grupo 1 foram agrupados em cinco duplas e os 11 do grupo 2, em 4 duplas e um trio. Cabe ressaltar que todos esses licenciandos já haviam participado de outro experimento com tablets, na disciplina considerada, focalizando a interpretação geométrica de sistemas lineares.

Antecendo o uso do GeoGebra no tablet, os licenciandos realizaram atividades com esse software no computador. Nas duas situações foram propostas atividades iniciais destinadas apenas à experimentação das ferramentas do GeoGebra. Tais atividades visavam orientar o uso dos recursos que seriam necessários nas atividades. No computador, a versão utilizada foi a Java Webstart 4.2.60.0 e, no tablet, a 4.3.27.1.

Para o estudo de caso, foram organizados dois encontros, promovidos em horário regular da disciplina, ambos com duração de $2 \mathrm{~h} 30 \mathrm{~min}$. O primeiro teve em vista as seguintes ações: i) discutir o significado da expressão "Geometria Dinâmica", a partir da leitura de um artigo; ii) experimentar as ferramentas do GeoGebra no computador; iii) resolver cinco atividades de Geometria Plana.

O segundo encontro teve como objetivo experimentar e analisar os recursos do GeoGebra em tablets com sistema operacional Android. Para tanto, foram propostas cinco atividades de Geometria Plana, distintas das realizadas no primeiro encontro. A proposta da resolução das atividades, nos dois encontros, visou possibilitar o reconhecimento dos recursos do GeoGebra associado à construção de conhecimentos geométricos. Assim, foi possível destacar possibilidades pedagógicas do software.

Para a coleta de dados, foram adotados os seguintes instrumentos: observação, questionário e respostas das atividades. Buscou-se captar a percepção dos licenciandos quanto ao uso pedagógico do GeoGebra no computador e no tablet. Os dados foram analisados segundo uma abordagem, predominantemente, qualitativa.

Ações e comentários feitos durante as aulas eram registrados para posterior análise. As atividades foram realizadas, pelos licenciandos, no GeoGebra, e as respostas foram registradas em editores de texto (tanto no computador quanto no tablet).

Em relação ao questionário, o mesmo continha perguntas abertas e fechadas, objetivando coletar dados que permitissem traçar um breve perfil dos participantes e analisar a visão dos mesmos sobre o uso do GeoGebra no computador e no tablet. Além disso, buscou-se investigar a facilidade de uso dos tablets institucionais e a importância do uso pedagógico de tablets, de maneira geral. Neste artigo, são analisados os dados considerados mais pertinentes ao relato.

\section{Análise e Discussão dos Resultados}

No primeiro encontro, foram promovidas as atividades mencionadas na seção 4. Os licenciandos manifestaram muito interesse pelo GeoGebra e testaram os recursos com facilidade, no computador. As orientações de uso das ferramentas contidas no próprio software (versão desktop) foram fundamentais para a experimentação das mesmas. Atribui-se essa importância ao fato de ser a primeira experiência desses licenciandos com um software de Geometria Dinâmica.

No segundo encontro, cada dupla ou trio recebeu um tablet. Os licenciandos participaram ativamente da resolução das atividades. Como já haviam utilizado o GeoGebra no computador, não ocorreram muitas dúvidas. Uma aluna expressou isso verbalmente e destacou também que, caso o GeoGebra fosse usado primeiro no tablet, ficaria tudo mais difícil, pois, no tablet não há orientações de uso das ferramentas. É 
importante minimizar as dificuldades durante a introdução de um software, pois os professores que se sentem confortáveis com a utilização de uma nova ferramenta, tendem a integrá-la mais facilmente em suas práticas docentes do que professores que tiveram muitos problemas iniciais (Hohenwarter J. et al., 2008). Ressalta-se que a falta das orientações na versão para tablets já havia sido percebida pelas pesquisadoras, porém não foi destacada na experimentação, pois o objetivo era possibilitar que os licenciandos identificassem essa e outras diferenças. Ainda durante a experimentação, outras duas alunas comentaram que preferiram usar o Geogebra no tablet e destacaram que a possibilidade de tocar e arrastar os entes geométricos pela tela transmitia uma sensação de proximidade, o que facilitava o estabelecimento de conjecturas. Como afirma Ciampa (2013), a interatividade e o feedback imediato, possibilitados pelo uso de recursos em tablets, podem contribuir para a curiosidade cognitiva dos alunos.

Ao final do segundo encontro, todos os sujeitos da pesquisa responderam ao questionário mencionado na seção anterior. Os resultados são discutidos a seguir. Dos 21 licenciandos, 17 eram do sexo feminino. A média de idade dos participantes era de 22 anos, com desvio padrão de, aproximadamente, quatro.

Quanto à utilização do GeoGebra no tablet e no computador, foram propostas cinco afirmativas diante das quais os 21 licenciandos deveriam assinalar uma das opções: Discordo Completamente (DC), Discordo (D), Não Concordo Nem Discordo (NC ND), Concordo (C) e Concordo Completamente (CC). A Tabela 1 mostra os resultados.

Tabela 1 - Utilização do GeoGebra

\begin{tabular}{|c|c|c|c|c|c|}
\hline & $\begin{array}{c}\text { DC } \\
\%\end{array}$ & $\begin{array}{l}\text { D } \\
\%\end{array}$ & $\begin{array}{l}\text { NC ND } \\
\%\end{array}$ & $\begin{array}{l}\mathrm{C} \\
\%\end{array}$ & $\begin{array}{c}\mathrm{CC} \\
\%\end{array}$ \\
\hline \multicolumn{6}{|c|}{ Afirmativa 1: A visualização das construções no aplicativo foi satisfatória. } \\
\hline Tablet & 9,5 & 4,8 & 9,5 & 38,1 & 38,1 \\
\hline Computador & 9,5 & 0 & 0 & 33,3 & 57,2 \\
\hline \multicolumn{6}{|c|}{ Afirmativa 2: Utilizar os recursos do software foi fácil. } \\
\hline Tablet & 9,5 & 14,3 & 23,8 & 28,6 & 23,8 \\
\hline Computador & 4,8 & 9,5 & 9,5 & 38,1 & 38,1 \\
\hline \multicolumn{6}{|c|}{ Afirmativa 3: O software oferece recursos que justificam sua utilização. } \\
\hline Tablet & 9,5 & 4,8 & 19,1 & 47,6 & 19,1 \\
\hline Computador & 9,5 & 0 & 9,5 & 33,3 & 47,7 \\
\hline \multicolumn{6}{|c|}{$\begin{array}{l}\text { Afirmativa 4: As representações das funções da interface (ícones, menus, etc.) são fáceis de } \\
\text { serem reconhecidas/entendidas. }\end{array}$} \\
\hline Tablet & 9,5 & 23,8 & 14,3 & 33,3 & 19,1 \\
\hline Computador & 9,5 & 0 & 19,1 & 38,1 & 33,3 \\
\hline \multicolumn{6}{|c|}{$\begin{array}{l}\text { Afirmativa 5: A utilização do GeoGebra no computador contribuiu para a realização da } \\
\text { atividade promovida. }\end{array}$} \\
\hline Tablet & 9,5 & 4,8 & 14,3 & 38,1 & 33,3 \\
\hline Computador & 4,8 & 4,8 & 9,5 & 23,8 & 57,1 \\
\hline
\end{tabular}

Considerando o somatório da opção Concordo com a Concordo Completamente, o uso do GeoGebra no computador foi melhor avaliado em todas as afirmativas propostas. Atribui-se esse resultado à maior familiaridade com programas de computador e às restrições da versão para tablets, por ser ainda muito nova. É importante considerar ainda que, como os tablets não eram dos licenciandos, não houve possibilidade de investigação dos recursos fora do tempo da aula, como fizeram no computador. Nesse sentido, Mang e Wardley (2012) defendem que é essencial que os alunos utilizem os dispositivos regularmente nas atividades didáticas, para que uso educacional dos tablets se torne natural. Uma pergunta aberta solicitando comentários sobre o GeoGebra no 
tablet permitiu levantar opiniões dos licenciandos que justificam a visão mais favorável ao computador, como as duas apresentadas abaixo:

O Geogebra é definitivamente um software para ser utilizado no desktop. Faz muita falta um "botão direito" nele. Outro ponto negativo foi a falta das instruções de uso das ferramentas quando o mouse é "passado" sobre elas (Aluno C).

é muito bom ser utilizado, por causa da mobilidade, etc. Mas me incomodou um pouco o fato da tela ser reduzida e a falta das explicações dos ícones o que dificulta um pouco a utilização para aqueles que não usaram o geogebra no computador e não conhecem a função dos ícones (Aluno E).

Destacam-se dois comentários quanto ao uso do GeoGebra no computador, são eles:

Fantástico! A interface é muito intuitiva, e inclusive já o utilizei para preparar uma aula sem grandes problemas (Aluno $C$ ).

no computador é muito bom porque ali se voce ficar perdido ele tem o ajuda e tem instruções é bem melhor (Aluno J).

Quando questionados sobre o uso dos tablets, de maneira geral, a maioria respondeu que foi fácil ou muito fácil (81\%). O aluno I considerou regular pela falta de costume de usar o dispositivo, já o aluno $\mathrm{K}$ destacou que será necessário tempo para a adaptação. Cabe ressaltar que os tablets utilizados são institucionais e não eram usados pelos participantes constantemente. Assim, entende-se que a falta de prática pode ter contribuído para algum desconforto no manuseio dos recursos do GeoGebra e, consequentemente, influenciado na avaliação do mesmo.

Embora o GeoGebra tenha sido melhor avaliado no computador, a maioria dos licenciandos considerou, como futuro professor, que os tablets podem ser bons recursos pedagógicos. Uma pergunta aberta possibilitou captar justificativas para esta afirmação. Abaixo, destacam-se duas delas. Nas mesmas, a importância de utilizar, no contexto educacional, tecnologias que são usadas no dia a dia são mencionadas como forma de despertar o interesse dos alunos:

Sim, pois além da mobilidade e a boa visualização dos gráficos, é um ótimo estimulador na aprendizagem, pois os alunos gostam de utilizar esses recursos tecnológicos (Aluno E).

Sim. pois alem de facilitar o estudo da materia aproxima a materia da realidade do aluno (Aluno $Q$ ).

Outros aspectos foram destacados, ainda nesta questão, quanto ao uso pedagógico de tablets, são eles: mobilidade, necessidade do professor ser capacitado, limitação dos aplicativos em relação aos softwares equivalentes utilizados no computador, o fato do tablet ser mais leve do que notebooks e a possibilidade de tornar a aula mais dinâmica Os comentários desta questão reforçam os resultados encontrados na pesquisa realizada por Goodwin (2012), como destacado na seção 2.

As respostas das atividades foram corrigidas e o resultado foi muito satisfatório. Vale ressaltar que alguns conteúdos ainda não haviam sido estudados na licenciatura. Uma aluna comentou que a partir das atividades realizadas conseguiu compreender o tema "pontos notáveis do triângulo", assunto que faria parte da avaliação da disciplina Geometria I. O uso GeoGebra, como discutido na seção 3, possibilita investigações e levantamento de hipóteses, o que favorece a aprendizagem.

\section{Considerações finais}

O GeoGebra é um software rico em possibilidades educacionais. A evolução do mesmo, ao longo dos anos, é algo que merece destaque. Trata-se de um esforço conjunto do seu desenvolvedor e de inúmeros membros da comunidade acadêmica, de diversos países, para torná-lo constantemente melhor e acessível em diferentes plataformas.

A proposta pedagógica associada ao GeoGebra requer incentivo à investigações, descobertas, discussões e reflexões. Assim, o uso desse software pode até mesmo ser 
considerado difícil, ou não muito adequado, por professores mais tradicionais. Nesse sentido, o trabalho com o GeoGebra, ou com outros softwares com propostas semelhantes, é fundamental nas licenciaturas em Matemática, tendo em vista a formação de profissionais com posturas mais abertas a estratégias de construção de conhecimento.

Particularmente, em relação à versão para tablets, é fundamental ressaltar que a mesma é bastante recente e, portanto, ainda em pleno desenvolvimento. Ainda assim, na pesquisa promovida, a versão para sistemas Android foi avaliada de forma positiva pela maioria dos participantes.

A versão para computador foi melhor avaliada, mas, nessa avaliação, é preciso considerar, além da fase inicial da versão para tablets, a maior familiaridade dos participantes com o computador e, até mesmo, características do equipamento utilizado.

Apesar de algumas dificuldades relatadas, cabe destacar que o potencial dos tablets para uso pedagógico foi evidenciado. A motivação e participação dos alunos são aspectos muito positivos. Porém, certamente, como qualquer outro recurso, esses dispositivos precisam ser entendidos como instrumentos mediadores da aprendizagem e utilizados sempre com objetivos pedagógicos bem definidos.

Como estudos futuros, pretende-se realizar atividades semelhantes às descritas neste artigo, com outros grupos de licenciandos e com professores em serviço.

\section{Notas de Texto}

1 <http://www.geogebra.org/cms/pt_BR/download/>; <http://web.geogebra.org>.

2 O GeoGebra Java Webstart, segundo informações do fórum oficial do software (http://www.geogebra.org/forum/viewtopic.php?f=56\&t=32201) será descontinuado em breve.

${ }^{3}<\mathrm{http}: / /$ www.geogebratube.org/>.

${ }^{4}$ Desenvolvido nas licenciaturas da instituição em questão, desde 2010, tendo como objetivo geral implementar ações direcionadas à formação de professores.

\section{Referências Bibliográficas}

CHAURASIA, P. GeoGebra Enabled Mathematics Curriculum - A Future Perspective. 2013. Disponível em <http://blog.geogebra.org/2013/06/geogebra-enabledmathematics-curriculum-a-future-perspective/>. Acesso em: 03 out. 2013.

CIAMPA, K. Learning in a mobile age: an investigation of student motivation. Journal of Computer Assisted Learning, p. 1-15, Aug. 2013.

CLARKE, B; SVANAES, S; ZIMMERMANN, S. One-to-one Tablets in Secondary Schools: an evaluation study. Stage 2: January - April 2013. Family Kids and Youth, 2013. Disponível em: <http://www.tabletsforschools.co.uk/wpcontent/uploads/2012/12/FKY-Tablets-for-Schools-Stage-2-Full-Report-July2013.pdf>. Acesso em: 4 out. 2013.

EDWARD, J.; JONES, K. Linking Geometry and Algebra with GeoGebra. Mathematics Teaching, v. 194, p. 28-30, 2006.

GOODWIN, K. Use of tablet technology in the classroom. 2012. Disponível em: <http://rde.nsw.edu.au/files/iPad_Evaluation_Sydney_Region_exec_sum.pdf $>$. Acesso em: 03 out. 2013.

HOHENWARTER, M.; FUCHS, K. Combination of dynamic geometry, algebra and calculus in the software system GeoGebra. In: COMPUTER ALGEBRA SYSTEMS AND DYNAMIC GEOMETRY SYSTEMS IN MATHEMATICS TEACHING CONFERENCE, 2004. Pécs, Hungary, Proceedings. Disponível em: <http://www.geogebra.org/publications/pecs_2004.pdf> Acesso em: 03 out. 2013.

HOHENWARTER, M. GeoGebra - didaktische Materialien und Anwendungen für den Mathematikunterricht. PhD thesis, University of Salzburg, Salzburg. 2006. 
Disponível em: <http://www.geogebra.org/publications/mhohen_diss.pdf> Acesso em: 03 out. 2013.

HOHENWARTER, M.; LAVICZA, Z. Mathematics teacher development with ICT: Towards an International GeoGebra Institute. In: KÜCHEMANN, D. (Ed.), Proceedings of the British Society for Research into Learning Mathematics, University of Northampton, UK, BSRLM, v. 27, p. 49-54, 2007.

HOHENWARTER, M.; PREINER, J. Dynamic mathematics with GeoGebra. The Journal of Online Mathematics and its Applications, v.7, 2007. Disponível em: <http://www.maa.org/external_archive/joma/Volume7/Hohenwarter/index.html>.

Acesso em: 03 out. 2013.

HOHENWARTER, J.; HOHENWARTER, M.; LAVICZA, Z. Introducing Dynamic Mathematics Software to Secondary School Teachers: The Case of GeoGebra. Journal of Computers in Mathematics and Science Teaching, v. 28, n.2, p. 135-146, 2008.

HOHENWARTER, M. GeoGebra 4.4 - from Desktops to Tablets. Indagatio Didactica, v. 5, n. 1, p. 8-18, 2013a.

HOHENWARTER, M. GeoGebra Tablet Apps. 2013b. Disponível em: <http://blog.geogebra.org/2013/09/geogebra-tablet-apps/>. Acesso em: 03 out. 2013.

KEARNEY, M; MAHER, D. Mobile learning in Maths teacher education: using ipads to support pre-service teachers' professional development. Australian Educational Computing. v. 27, n. 3, p. 76-84, 2013.

KOEHLER, M. J.; MISHRA, P. What is technological pedagogical content knowledge? Contemporary Issues in Technology and Teacher Education,v.9, n. 1, p.60-70, 2009. LJAJKO, E. Development of ideas in a GeoGebra - aided mathematics instruction. Mevlana International Journal of Education (MIJE). v. 3. 3 ed. Edição especial: Dynamic and Interactive Mathematics Learning Environments. p. 1-7, 2013.

MANG, C. F.; WARDLEY, L. J. Effective Adoption of Tablets in Post-Secondary Education: Recommendations Based on a Trial of iPads in University Classes. Journal of Information Technology Education: Innovations in Practice, EUA. v.11, p. 301317, 2012.

NASCIMENTO, A. C. T. A. de A. A Integração das Tecnologias às Práticas Escolares. In: Pesquisa sobre o uso das tecnologias de informação e comunicação no Brasil: TIC Educação 2012. São Paulo: Comitê Gestor da Internet no Brasil, 2013, p.45-49. Disponível em: <http://www.cetic.br/publicacoes/2012/tic-educacao-2012.pdf>. Acesso em: 11 out. 2013.

PREINER, J. Introducing Dynamic Mathematics Software to Mathematics Teachers: the Case of GeoGebra. Faculty of Natural Sciences University of Salzburg, Salzburg, 2008. Disponível em: <http://www.geogebra.org/publications/jpreinerdissertation.pdf> Acesso em: 03 out. 2013.

SEABRA, C. Tablets na sala de aula. 2012. Disponível em: <http://cseabra.wordpress.com/2012/04/22/tablets-na-sala-de-aula/>. Acesso em: 04 out. 2013.

SHULMAN L. S. Those Who Understand: Knowledge Growth in Teaching. Educational Researcher, v. 15, n. 2, p. 4-14, Feb.1986.

ZAKARIA, E.; LEE, L. S. Teachers' Perceptions toward the use of GeoGebra in the Teaching and Learning of Mathematics. Journal of Mathematics and Statistics, Austrália. v. 8, n. 2, p. 253-257, 2012. 